\title{
Universal Projective Synchronization of Two Different Hyperchaotic Systems with Unknown Parameters
}

\author{
Baojie Zhang ${ }^{1,2}$ and Hongxing $\mathrm{Li}^{1}$ \\ ${ }^{1}$ Faculty of Electronic Information and Electrical Engineering, Dalian University of Technology, Dalian 116024, China \\ ${ }^{2}$ School of Mathematics and Information Science, Qujing Normal University, Qujing 655011, China \\ Correspondence should be addressed to Baojie Zhang; qjupk123@mail.dlut.edu.cn
}

Received 8 November 2013; Accepted 10 January 2014; Published 12 March 2014

Academic Editor: Li Weili

Copyright (c) 2014 B. Zhang and H. Li. This is an open access article distributed under the Creative Commons Attribution License, which permits unrestricted use, distribution, and reproduction in any medium, provided the original work is properly cited.

Universal projective synchronization (UPS) of two chaotic systems is defined. Based on the Lyapunov stability theory, an adaptive control method is derived such that UPS of two different hyperchaotic systems with unknown parameters is realized, which is up to a scaling function matrix and three kinds of reference systems, respectively. Numerical simulations are used to verify the effectiveness of the scheme.

\section{Introduction}

Chaotic synchronization has gained extensively and intensively attention in recent decades since Pecora and Carroll's pioneering and meaningful work [1]. It has applications in vast areas of physics, chemistry, biology, and others particularly in secure communication. Various synchronization modes are proposed, such as complete synchronization [1], phase synchronization [2], projective synchronization [3], and antisynchronization [4]. Projective synchronization (PS) [5] as a generalization of complete synchronization (CS) and antisynchronization (AS), has attracted much attention for the scaling factor in which the state vectors become proportional.

The projective synchronization is firstly applied in a class of systems with partial linearity. Yan and Li [6] applied this notion in a general class of chaotic systems including nonpartially-linear systems in 2005: this synchronization mode was called generalized projective synchronization (GPS). In [7], the modified projective synchronization (MPS) is presented to synchronize two identical systems up to a scaling constant matrix. In 2007, Chen and Li [8] gave a new synchronization mode-function projective synchronization (FPS) in which scaling function matrix substitutes the scaling constant matrix. Tang et al. [9] presented FPS in another familiar way by using a function instead of the scaling factor of GPS. Du et al. [10] applied Tang's definition to synchronize different chaotic systems with uncertain parameters. A more general definition called modified function projective synchronization (MFPS) was given by Du et al. [11] in 1999 and was used in secure communication. In recent years, many efforts are concentrated on MFPS [12-14].

From the development, we know that the scaling factor is expanded from constant to constant matrix and from function to matrix function.

In this paper, we will introduce a new type of synchronization called universal projective synchronization (UPS) which concerns the displacement. Adaptive synchronization is an effective method for dealing with unknown parameters $[10,15]$. Zhang et al. [16] proposed a new hyperchaotic system. UPS of hyperchaotic L $\ddot{u}$ system [17] and the new one with unknown parameters will be investigated by using adaptive control method. Three kinds of reference systems are chosen for the UPS. The introduction of UPS will expand the scope of synchronization.

The rest of this paper is organized as follows. In Section 2, the definition of UPS is given. In Section 3, the UPS of hyperchaotic L $\ddot{u}$ system and the new one is studied. Adaptive control schemes are proposed. Simulation results verify the effectiveness of the schemes. The conclusion is given in Section 4. 


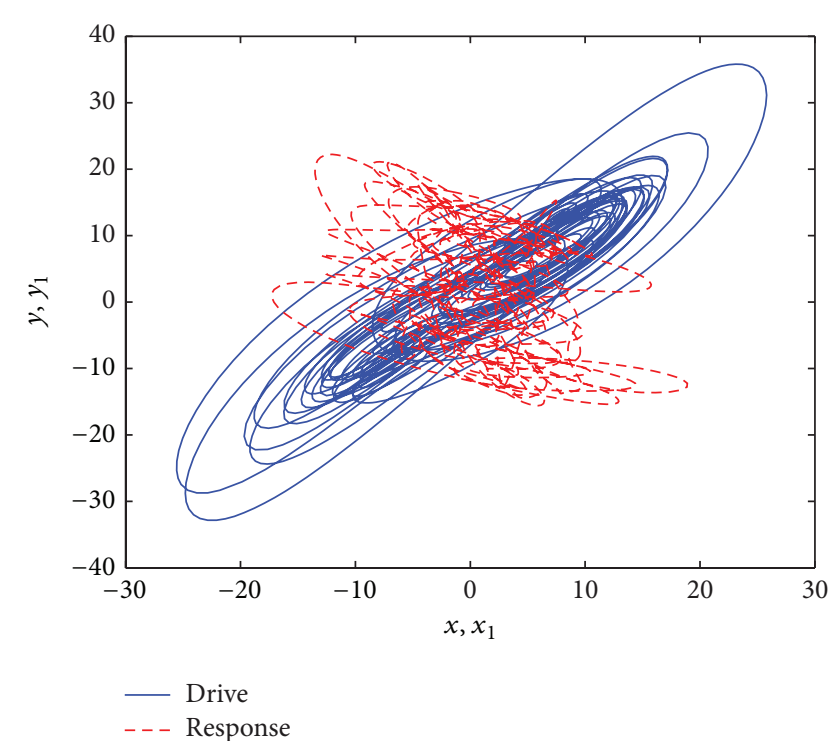

FIGURE 1: The trajectories projection on $x-y\left(x_{1}-y_{1}\right)$ plane of the drive system and the response system.

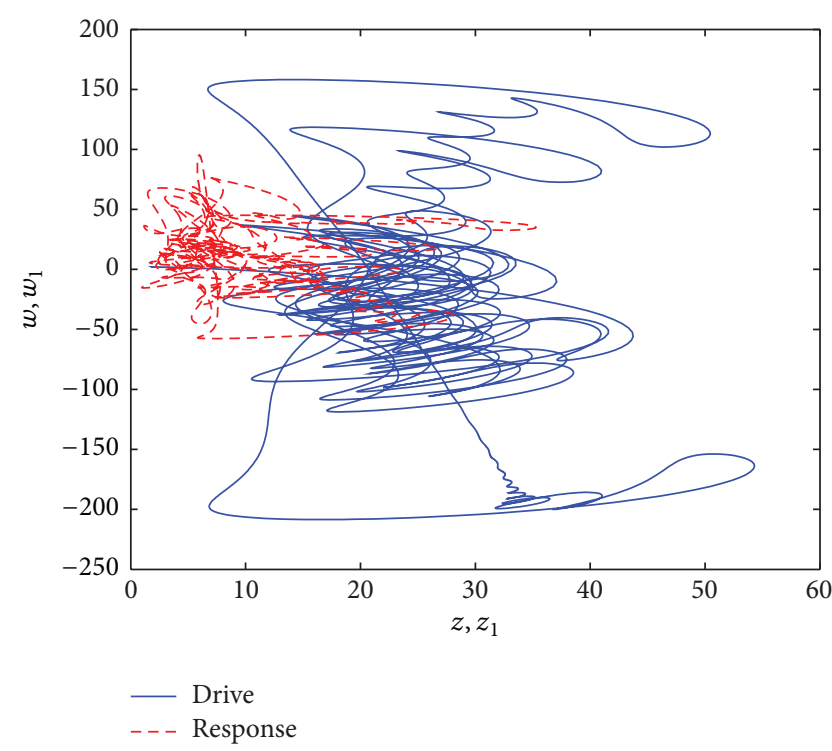

FIGURE 2: The trajectories projection on $z-w\left(z_{1}-w_{1}\right)$ plane of the drive system and the response system.

\section{The Definition of UPS}

The drive system and the response system are defined as follows:

$$
\begin{gathered}
\dot{\mathbf{x}}=\mathbf{f}(t, \mathbf{x}), \\
\dot{\mathbf{y}}=\mathbf{g}(t, \mathbf{y})+\mathbf{u}(t, \mathbf{x}, \mathbf{y}),
\end{gathered}
$$

where $\mathbf{x}=\left(x_{1}, x_{2}, \ldots, x_{n}\right)^{T}$ and $\mathbf{y}=\left(y_{1}, y_{2}, \ldots, y_{n}\right)^{T} \epsilon$ $R^{n}$ are the state vectors; $\mathbf{f}, \mathbf{g}: R^{n} \rightarrow R^{n}$ are continuous nonlinear vector function; $\mathbf{u}(t, \mathbf{x}, \mathbf{y}) \in R^{n}$ is the controller to

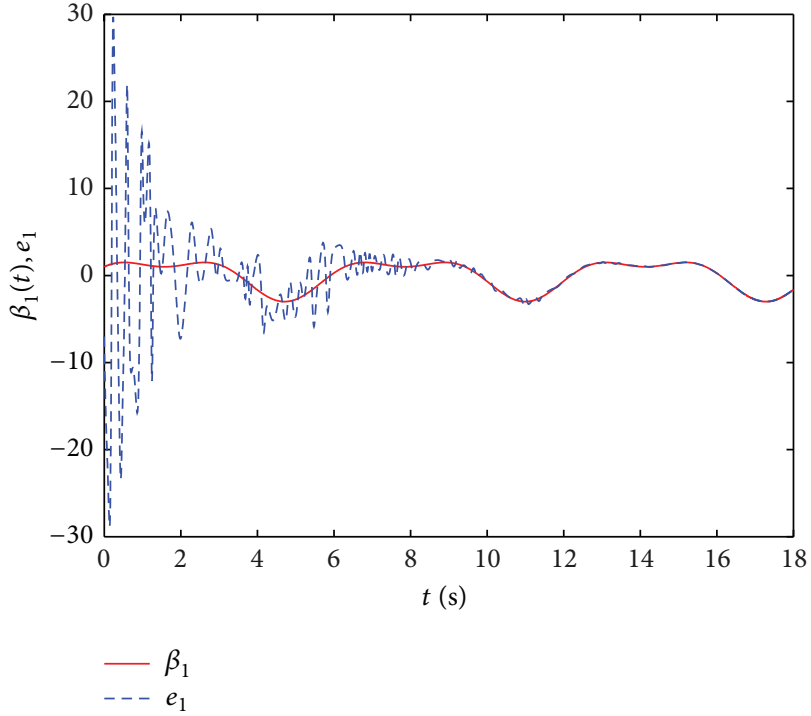

Figure 3: The time evolution of the error $e_{1}$ and the function $\beta_{1}(t)$.

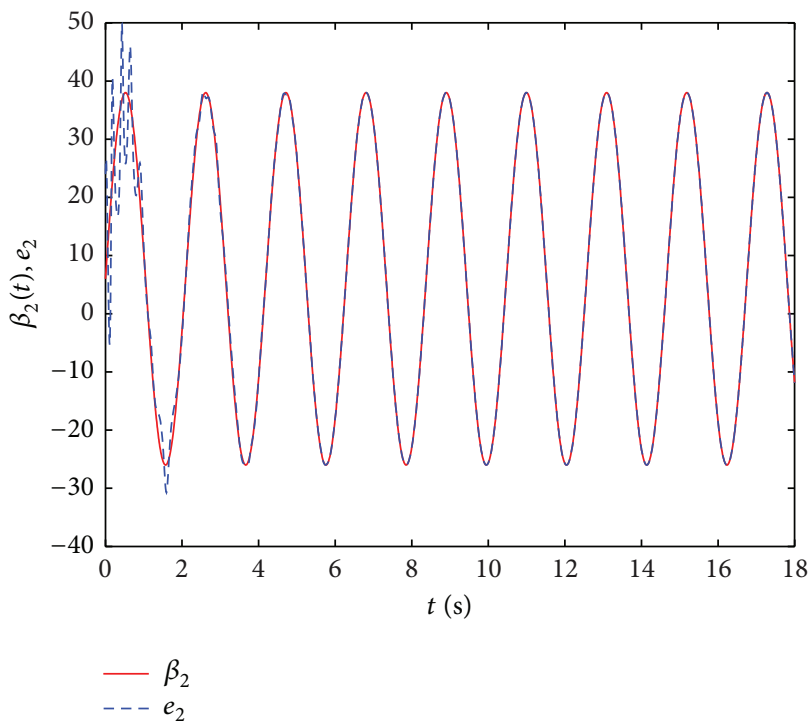

FIGURE 4: The time evolution of the error $e_{2}$ and the function $\beta_{2}(t)$.

be designed. If there is no controller $\mathbf{u}(t, \mathbf{x}, \mathbf{y}),(1)$ and (2) are chaotic systems. We define the state error vector

$$
\mathbf{e}=\left(e_{1}, e_{2}, \ldots, e_{n}\right)^{T} \triangleq \mathbf{x}-\mathbf{A}(t) \mathbf{y}
$$

where $\mathbf{A}(t)=\operatorname{diag}\left(\alpha_{1}(t), \alpha_{2}(t), \ldots, \alpha_{n}(t)\right) ; \alpha_{i}(t)(i=$ $1,2,3, \ldots, n)$ is continuous differentiable function with boundness, and $\alpha_{i}(t) \neq 0$ for all $t$. Obviously, the error system is

$$
\dot{\mathbf{e}}=\mathbf{f}(t, \mathbf{x})-\dot{\mathbf{A}}(t) \mathbf{y}-\mathbf{A}(t)(\mathbf{g}(t, \mathbf{y})+\mathbf{u}(t, \mathbf{x}, \mathbf{y})) .
$$

Suppose there exists a reference system

$$
\dot{\mathbf{z}}=\mathbf{h}(t, \mathbf{z}),
$$




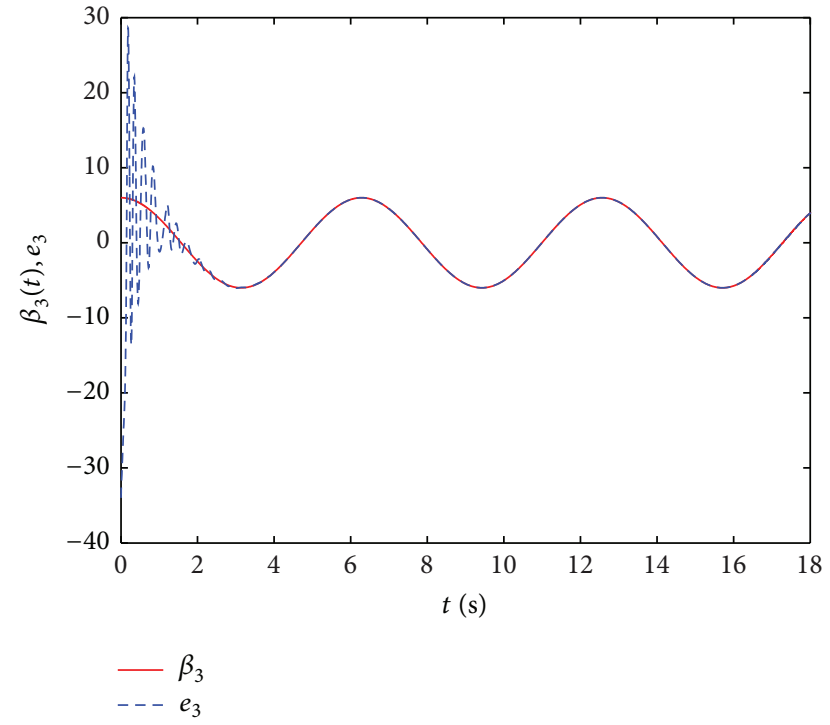

FIGURE 5: The time evolution of the error $e_{3}$ and the function $\beta_{3}(t)$.

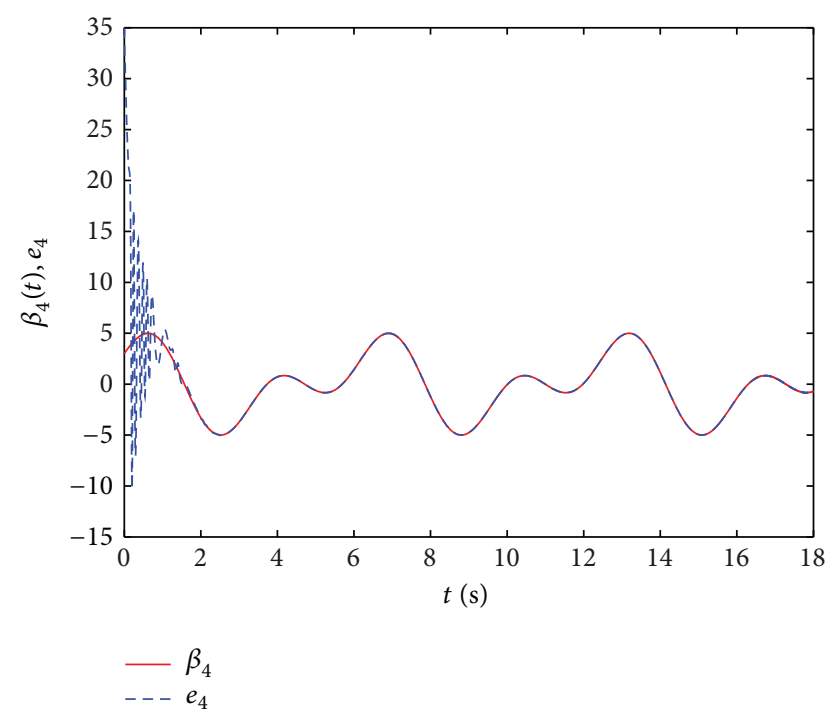

FIgURE 6: The time evolution of the error $e_{4}$ and the function $\beta_{4}(t)$.

where $\mathbf{z}=\left(z_{1}, z_{2}, \ldots, z_{n}\right)^{T} \in R^{n}, \mathbf{h}: R^{n} \rightarrow R^{n}$ is a continuous differentiable function. Suppose the reference system is an attractor.

Definition 1. For the scaling diagonal matrix function $\mathbf{A}(t)$, it is said that the drive system (1) and the response system (2) are universal projective synchronization (UPS) in the sense of the system (5), if the error system (4) and the reference system (5) are completely synchronized; that is,

$$
\lim _{t \rightarrow \infty}\|\mathbf{e}-\mathbf{z}\|=0 .
$$

Now we give another dual definition of UPS. We define the state error vector

$$
\mathbf{e}^{*}=\left(e_{1}^{*}, e_{2}^{*}, \ldots, e_{n}^{*}\right)^{T} \triangleq \mathbf{A}(t) \mathbf{x}-\mathbf{y}
$$

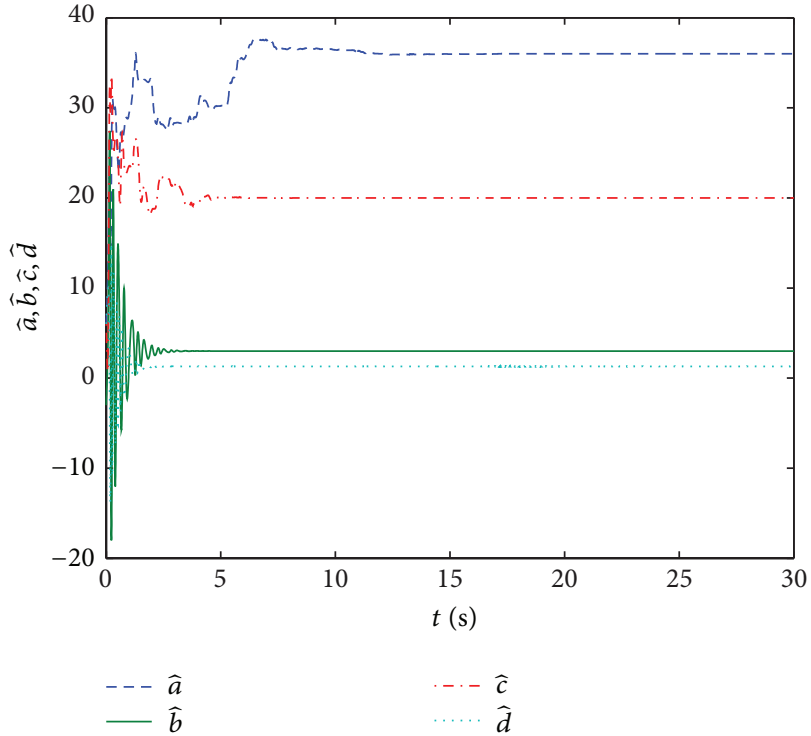

FIgURE 7: The time evolution of the estimated values $\widehat{a}, \widehat{b}, \widehat{c}$, and $\widehat{d}$.

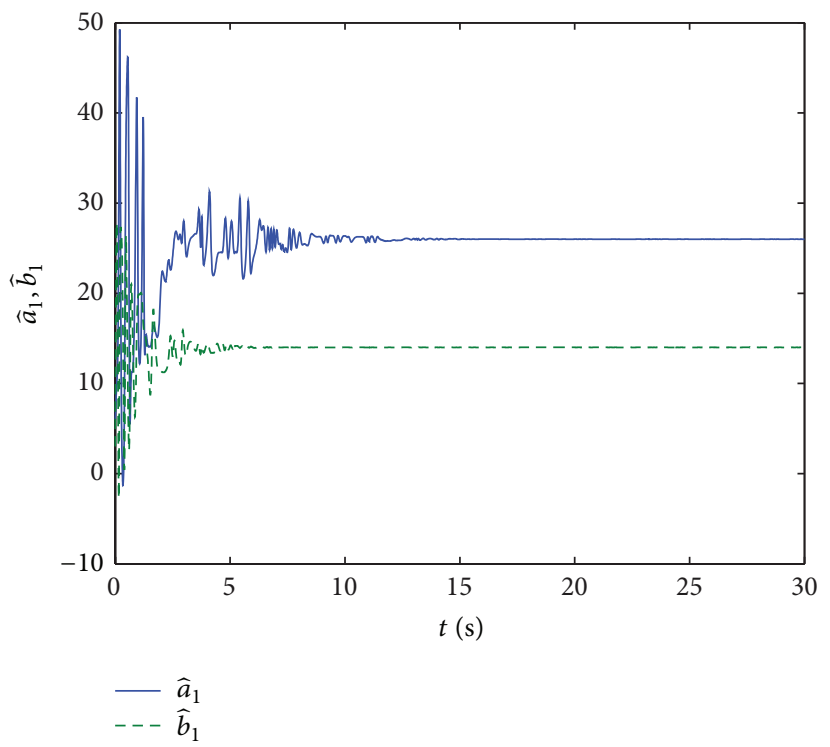

FIGURE 8: The time evolution of the estimated values $\widehat{a}_{1}$ and $\widehat{b}_{1}$.

then the error system is

$$
\dot{\mathbf{e}}^{*}=\dot{\mathbf{A}}(t) \mathbf{x}+\mathbf{A}(t) \mathbf{f}(t, \mathbf{x})-\mathbf{g}(t, \mathbf{y})-\mathbf{u}(t, \mathbf{x}, \mathbf{y}) .
$$

Definition 2. For the scaling diagonal matrix function $\mathbf{A}(t)$, it is said that the drive system (1) and the response system (2) are universal projective synchronization (UPS) in the sense of the system (5), if there exists a scaling diagonal matrix function $\mathbf{A}(t)$ such that the error system (8) and the reference system (5) are completely synchronized; that is,

$$
\lim _{t \rightarrow \infty}\left\|\mathbf{e}^{*}-\mathbf{z}\right\|=0 .
$$




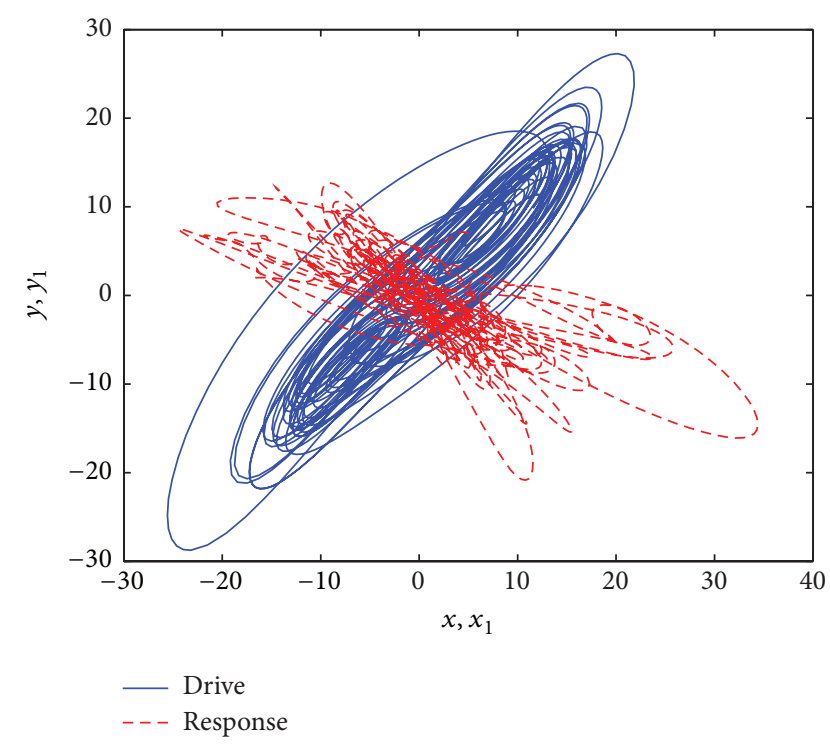

Figure 9: The trajectories projection on $x-y\left(x_{1}-y_{1}\right)$ plane of the drive system and the response system.

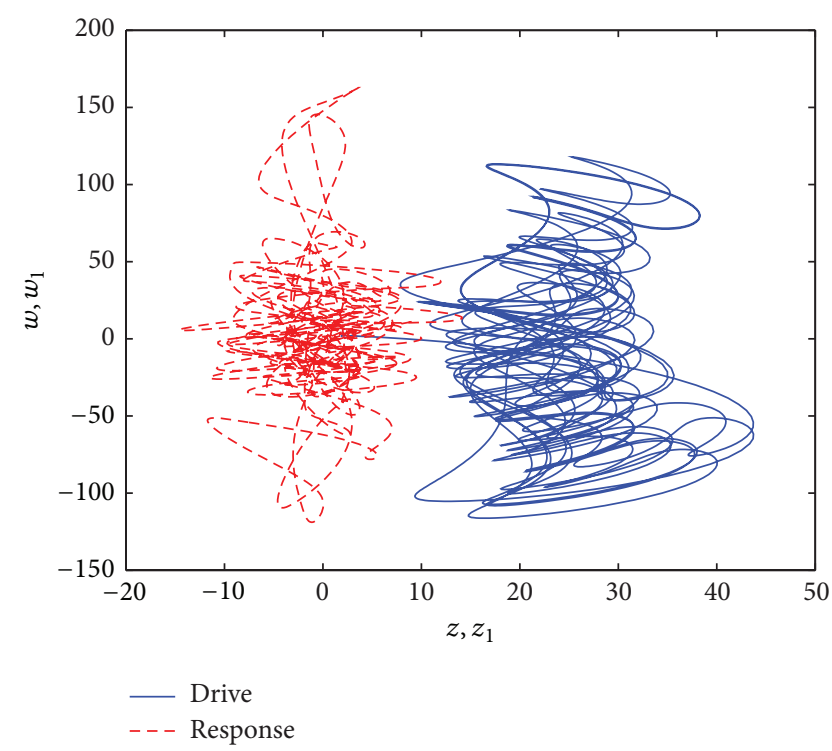

FIGURE 10: The trajectories projection on $z-w\left(z_{1}-w_{1}\right)$ plane of the drive system and the response system.

Remark 3. It is easy to see that UPS degenerated to MFPS if the reference system denotes constant vector $\mathbf{0}$; that is, the initial value and state change rate of system (5) are all $\mathbf{0 .}$

Remark 4. The reference system (5) expresses a constant vector $\boldsymbol{\xi}$, if its initial value is $\boldsymbol{\xi}$ and the state change rate is $\mathbf{0}$. UPS is in the sense of constant vector $\xi$; that is, there exists a displacement $\xi$ between the drive system and response system. System (5) can be other attractors, such as periodic function, quasiperiod function, chaos, and hyperchaos.

Thereinafter UPS is always in the sense of Definition 1.

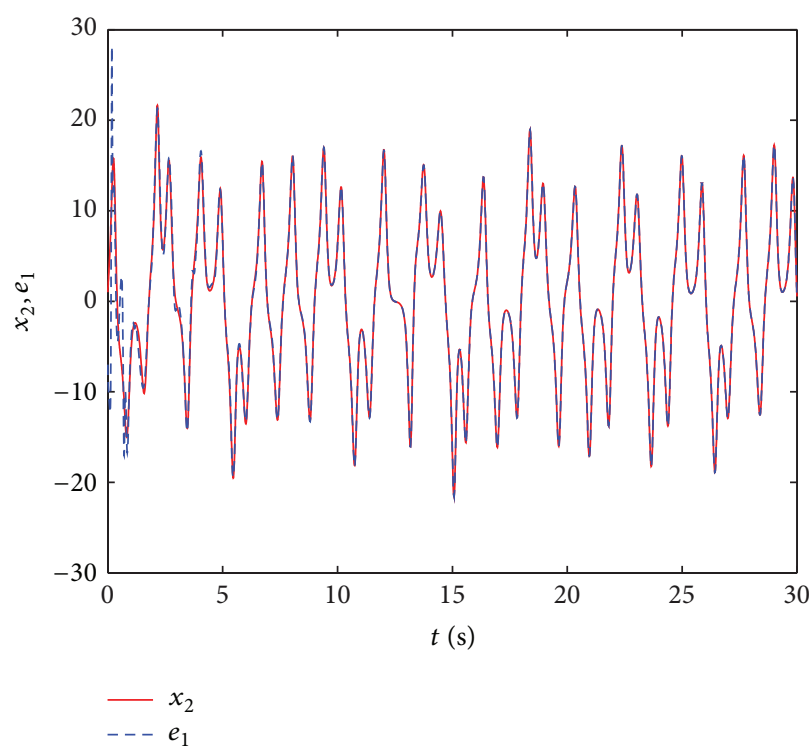

Figure 11: The time evolution of the error $e_{1}$ and the state $x_{2}$.

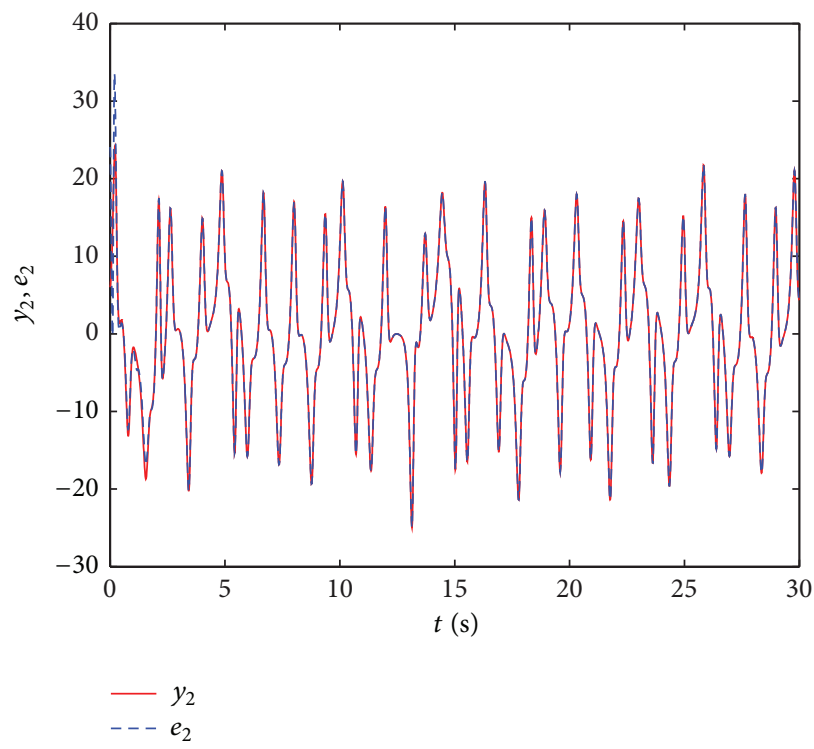

Figure 12: The time evolution of the error $e_{2}$ and the state $y_{2}$.

\section{UPS of Hyperchaotic Systems with Uncertain Parameters}

We take hyperchaotic L $\ddot{u}$ system [17] as the drive system, which is described by

$$
\begin{gathered}
\dot{x}=a(y-x)+w, \\
\dot{y}=-x z+c y, \\
\dot{z}=x y-b z, \\
\dot{w}=x z+d w,
\end{gathered}
$$




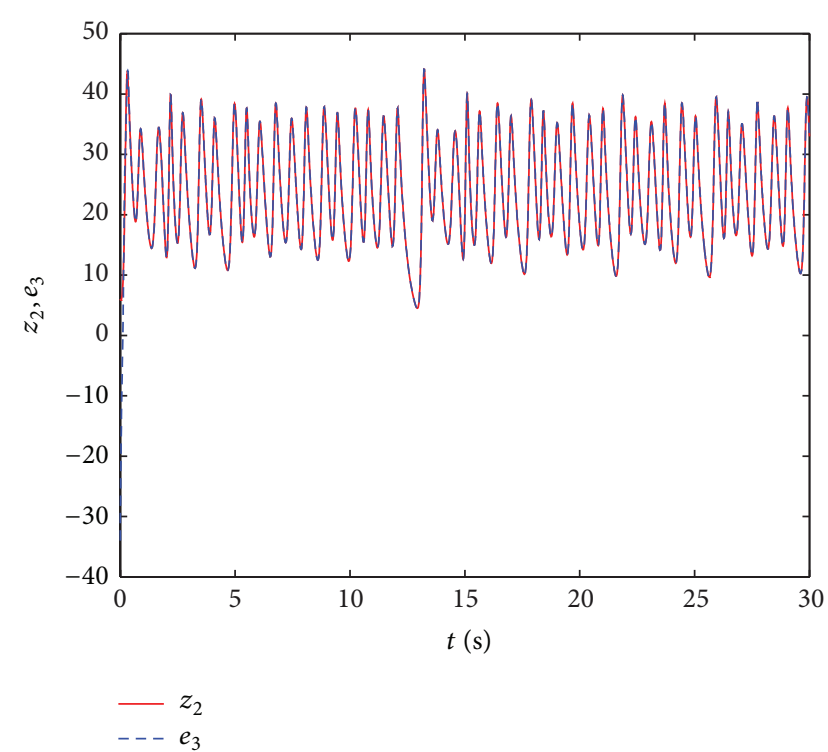

Figure 13: The time evolution of the error $e_{3}$ and the state $z_{2}$.

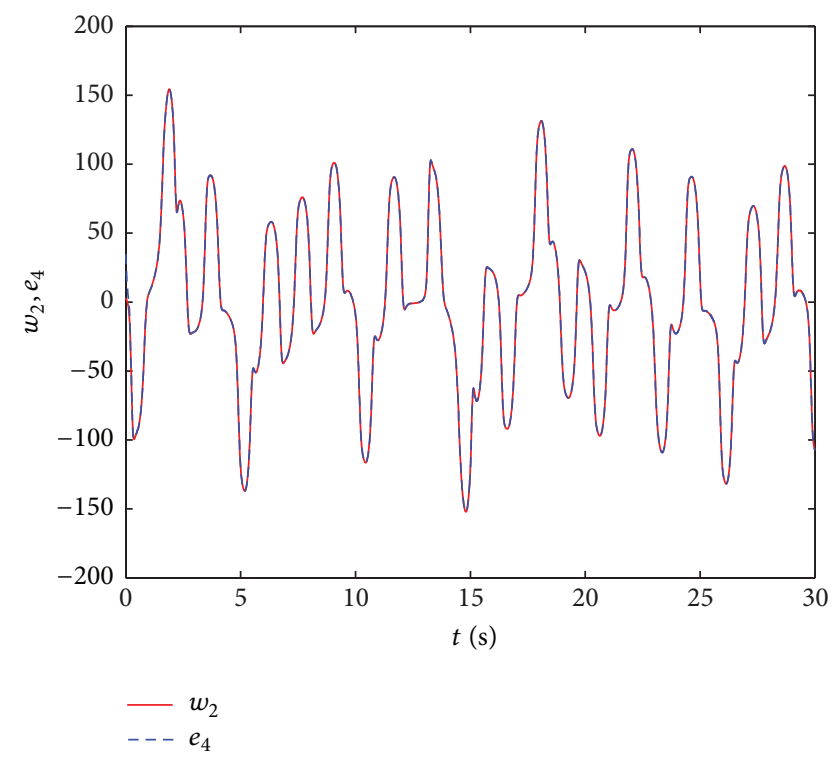

FIGURE 14: The time evolution of the error $e_{4}$ and the state $w_{2}$.

where $x, y, z, w$ are state variables and $a, b, c, d$ are uncertain parameters, which need to be estimated. The new hyperchaotic system presented by Zhang et al. [16], as the response system, is given by

$$
\begin{gathered}
\dot{x}_{1}=a_{1}\left(y_{1}-x_{1}\right)+u_{1}, \\
\dot{y}_{1}=b_{1} y_{1}-x_{1} z_{1}+w_{1}+u_{2}, \\
\dot{z}_{1}=x_{1} y_{1}-3.36 z_{1}+u_{3}, \\
\dot{w}_{1}=-x_{1} z_{1}+w_{1}+u_{4},
\end{gathered}
$$

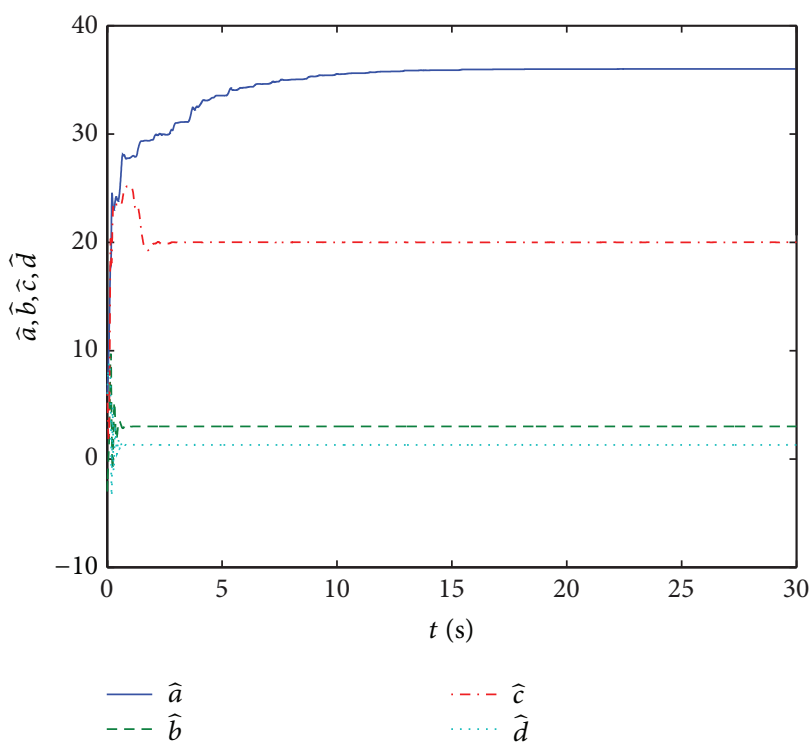

FIGURE 15: The time evolution of the estimated values $\widehat{a}, \widehat{b}, \widehat{c}$, and $\widehat{d}$.

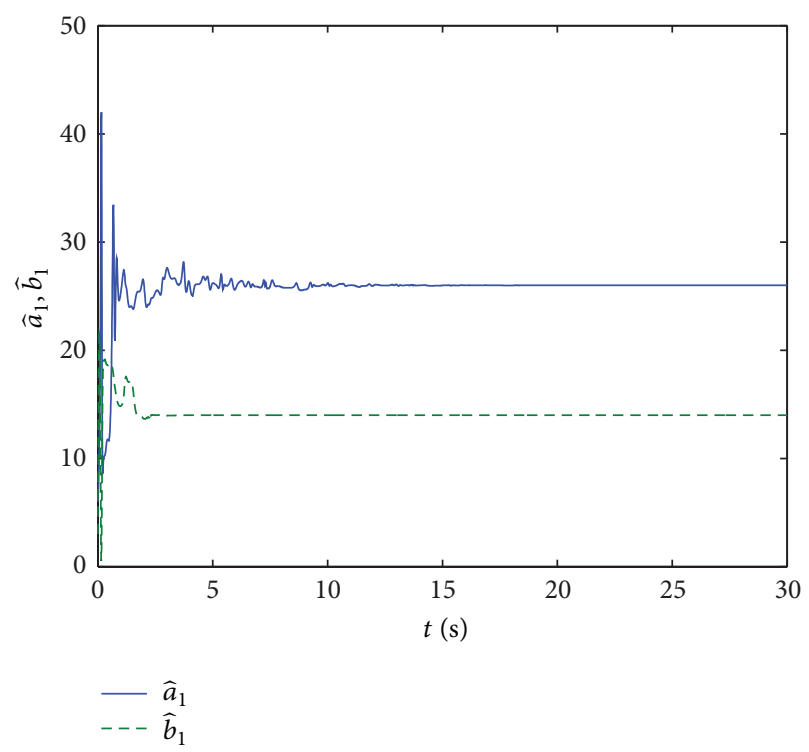

FIGURE 16: The time evolution of the estimated values $\widehat{a}_{1}$ and $\widehat{b}_{1}$.

where $x_{1}, y_{1}, z_{1}, w_{1}$ are state variables, $a_{1}, b_{1}$ are uncertain parameters, which need to be estimated, and $u_{1}, u_{2}, u_{3}, u_{4}$ are the nonlinear control laws. Suppose the scaling matrix $\mathbf{A}(t)=\operatorname{diag}\left(\alpha_{1}(t), \alpha_{2}(t), \alpha_{3}(t), \alpha_{4}(t)\right)$. Obviously the errors $e_{1}=x-\alpha_{1}(t) x_{1}, e_{2}=y-\alpha_{2}(t) y_{1}, e_{3}=z-\alpha_{3}(t) z_{1}$, and $e_{4}=w-\alpha_{4}(t) w_{1}$. The estimations of $a, b, c, d, a_{1}, b_{1}$ are $\widehat{a}, \widehat{b}, \widehat{c}, \widehat{d}, \widehat{a}_{1}, \widehat{b}_{1}$, respectively. $\widetilde{a}=\widehat{a}-a, \widetilde{b}=\widehat{b}-b, \widetilde{c}=\widehat{c}-c$, $\widetilde{d}=\widehat{d}-d, \widetilde{a}_{1}=\widehat{a}_{1}-a_{1}$, and $\widetilde{b}_{1}=\widehat{b}_{1}-b_{1}$ are the corresponding parameter estimate errors. 


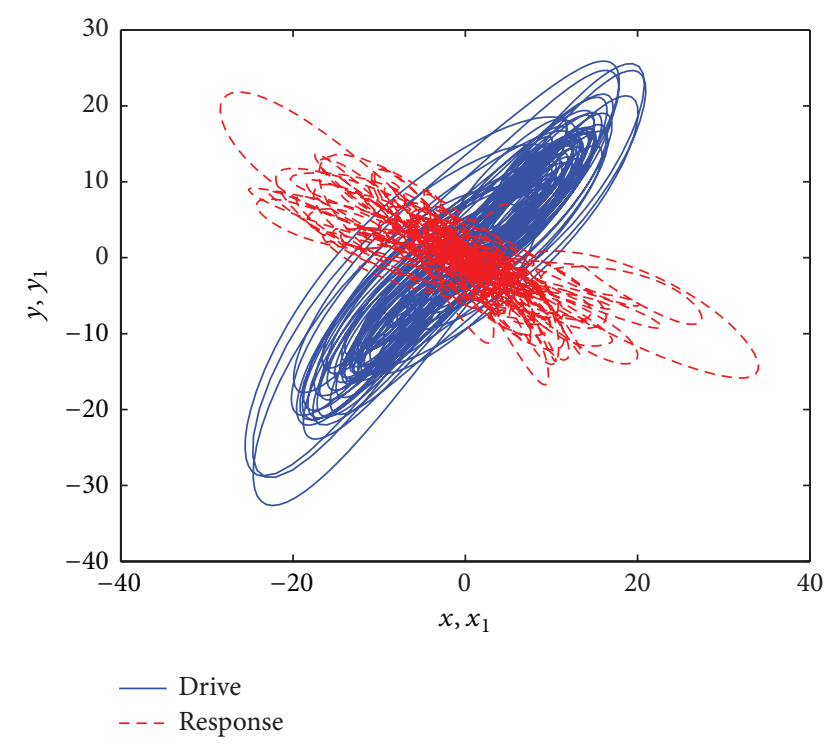

FIGURE 17: The trajectories projection on $x-y\left(x_{1}-y_{1}\right)$ plane of the drive system and the response system.

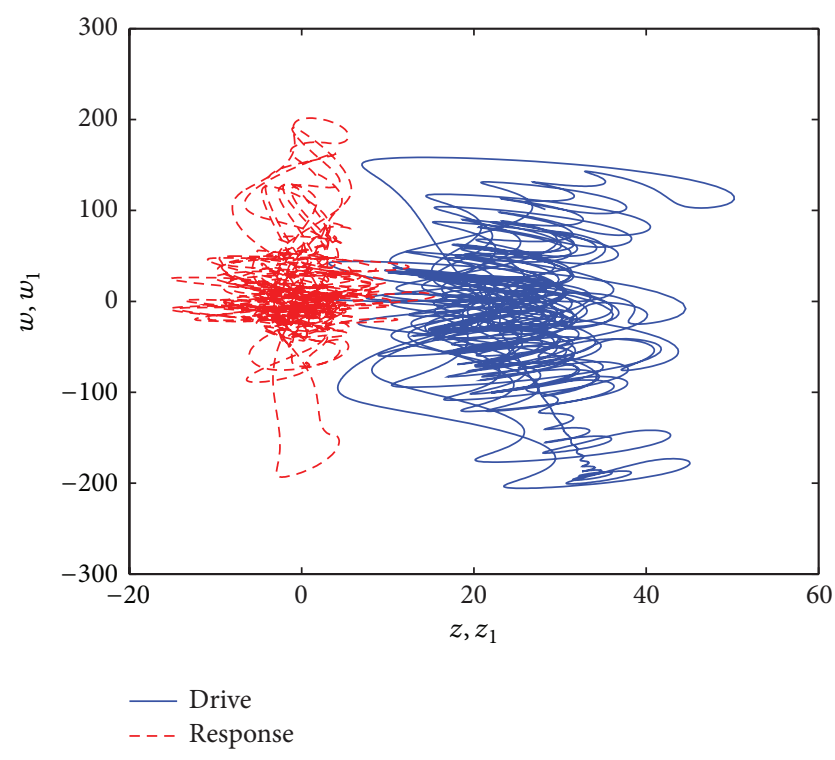

FIGURE 18: The trajectories projection on $z-w\left(z_{1}-w_{1}\right)$ plane of the drive system and the response system.

Now we discuss the UPS with three kinds of reference systems.

3.1. UPS in the Sense of Vector Function. Take the vector function $\boldsymbol{\beta}(t)=\left(\beta_{1}(t), \beta_{2}(t), \beta_{3}(t), \beta_{4}(t)\right)^{T}$ as the reference system. Let $\widetilde{\mathbf{e}}=\left(\widetilde{e}_{1}, \widetilde{e}_{2}, \widetilde{e}_{3}, \widetilde{e}_{4}\right)^{T} \triangleq\left(e_{1}-\beta_{1}, e_{2}-\beta_{2}, e_{3}-\beta_{3}, e_{4}-\right.$ $\left.\beta_{4}\right)^{T}$.

Theorem 5. For the given scaling matrix $\mathbf{A}(t)$ and the vector function $\boldsymbol{\beta}(t)$, the UPS between drive system (10) and response system (11) with unknown parameters will occur by the control $\mathbf{u}=\left(u_{1}, u_{2}, u_{3}, u_{4}\right)$ and parameter update law as follows:

$$
\begin{gathered}
u_{1}=\frac{1}{\alpha_{1}(t)}\left(\hat{a}(y-x)+w-\dot{\alpha}_{1}(t) x_{1}\right. \\
\left.-\alpha_{1}(t) \widehat{a}_{1}\left(y_{1}-x_{1}\right)-\dot{\beta}_{1}(t)+k_{1} \widetilde{e}_{1}\right), \\
u_{2}=\frac{1}{\alpha_{2}(t)}\left(-x z+\widehat{c} y-\dot{\alpha}_{2}(t) y_{1}+\alpha_{2}(t) x_{1} z_{1}\right. \\
\left.-\alpha_{2}(t) \hat{b} y_{1}-\alpha_{2}(t) w_{1}-\dot{\beta}_{2}(t)+k_{2} \widetilde{e}_{2}\right), \\
u_{3}=\frac{1}{\alpha_{3}(t)}\left(x y-\widehat{b} z-\dot{\alpha}_{3}(t) z_{1}-\alpha_{3}(t) x_{1} y_{1}\right. \\
\left.+3.36 \alpha_{3}(t) z_{1}-\dot{\beta}_{3}(t)+k_{3} \widetilde{e}_{3}\right), \\
u_{4}=\frac{1}{\alpha_{4}(t)}\left(x z+\hat{d} w-\dot{\alpha}_{4}(t) w_{1}+\alpha_{4}(t) x_{1} z_{1}\right. \\
\left.-\alpha_{4}(t) w_{1}-\dot{\beta}_{4}(t)+k_{4} \widetilde{e}_{4}\right), \\
\dot{\hat{a}}=(y-x) \tilde{e}_{1}, \\
\dot{\hat{b}}=-z \widetilde{e}_{3}, \\
\dot{\hat{c}}=y \widetilde{e}_{2}, \\
\dot{\hat{d}}=w \widetilde{e}_{4}, \\
\dot{\hat{a}}=-\alpha_{1}(t)\left(y_{1}-x_{1}\right) \widetilde{e}_{1}, \\
\dot{\vec{b}}=-\alpha_{2}(t) y_{1} \widetilde{e}_{2},
\end{gathered}
$$

where $k_{1}, k_{2}, k_{3}, k_{4}$ are positive control gains.

Proof. Choose the following Lyapunov function:

$$
V=\frac{1}{2}\left(\widetilde{\mathbf{e}}^{T} \widetilde{\mathbf{e}}+\widetilde{a}^{2}+\widetilde{b}^{2}+\widetilde{c}^{2}+\widetilde{d}^{2}+\widetilde{a}_{1}^{2}+\widetilde{b}_{1}^{2}\right) .
$$

Then the derivative of $V$ is given by

$$
\begin{aligned}
& \dot{V}=\frac{1}{2}\left(2 \widetilde{\mathbf{e}}^{T} \dot{\tilde{\mathbf{e}}}+2 \tilde{a} \dot{\vec{a}}+2 \tilde{b} \dot{\vec{b}}+2 \dot{\widetilde{c}}+2 \dot{\widetilde{d d}}\right. \\
& \left.+2 \widetilde{a}_{1} \dot{\tilde{a}}_{1}+2 \widetilde{b}_{1} \dot{\vec{b}}_{1}\right)
\end{aligned}
$$

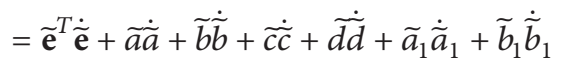

$$
\begin{aligned}
& =\widetilde{e}_{1}\left(a(y-x)+w-\dot{\alpha}_{1} x_{1}-\alpha_{1} a_{1}\left(y_{1}-x_{1}\right)\right. \\
& \left.-\alpha_{1} u_{1}-\dot{\beta}_{1}\right) \\
& +\widetilde{e}_{2}\left(-x z+c y-\dot{\alpha}_{2} y_{1}-\alpha_{2}\left(b_{1} y_{1}-x_{1} z_{1}+w_{1}\right)\right. \\
& \left.-\alpha_{2} u_{2}-\dot{\beta}_{2}\right) \\
& +\widetilde{e}_{3}\left(x y-b z-\dot{\alpha}_{3} z_{1}-\alpha_{3}\left(x_{1} y_{1}-c_{1} z_{1}\right)-\alpha_{3} u_{3}-\dot{\beta}_{3}\right) \\
& +\widetilde{e}_{4}\left(x z+d w-\dot{\alpha}_{4} w_{1}-\alpha_{4}\left(-x_{1} z_{1}+w_{1}\right)-\alpha_{4} u_{4}-\dot{\beta}_{4}\right) \\
& +\tilde{a} \dot{\vec{a}}+\tilde{b} \dot{\vec{b}}+\dot{\vec{c} c}+\tilde{\widetilde{d}}+\tilde{a}_{1} \dot{\tilde{a}}_{1}+\widetilde{b}_{1} \dot{\vec{b}}_{1}
\end{aligned}
$$




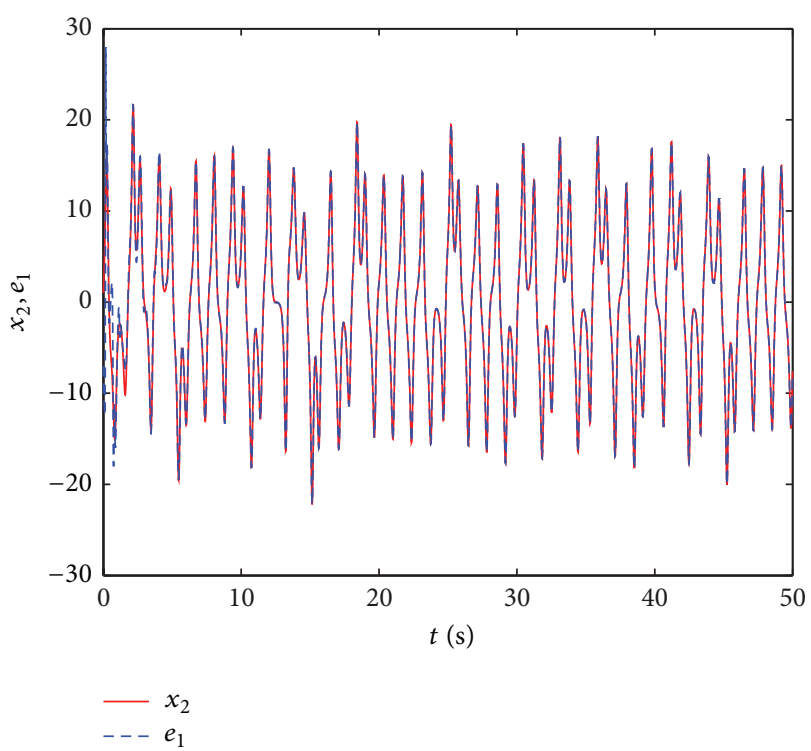

Figure 19: The time evolution of the error $e_{1}$ and the state $x_{2}$.

With the controls (12) and the parameter update laws (13), we have

$$
\dot{V}=-k_{1} \widetilde{e}_{1}^{2}-k_{2} \widetilde{e}_{2}^{2}-k_{3} \widetilde{e}_{3}^{2}-k_{4} \widetilde{e}_{4}^{2}
$$

Because $\dot{V}$ is negative simidefinite, $\widetilde{e}_{1}, \widetilde{e}_{2}, \widetilde{e}_{3}, \widetilde{e}_{4} \in L_{\infty}$, $\widetilde{a}, \widetilde{b}, \widetilde{c}, \widetilde{d}, \widetilde{a}_{1}, \widetilde{b}_{1} \in L_{\infty}$. From the definition of $\widetilde{\mathbf{e}}$, we know $\dot{\tilde{e}}_{1}, \dot{\tilde{e}}_{2}, \dot{\tilde{e}}_{3}, \dot{\tilde{e}}_{4} \in L_{\infty}$. From $\dot{V}=-k_{1} \tilde{e}_{1}^{2}-k_{2} \widetilde{e}_{2}^{2}-k_{3} \widetilde{e}_{3}^{2}-k_{4} \tilde{e}_{4}^{2}=$ $-\widetilde{e}^{T} K \widetilde{e}\left(K=\operatorname{diag}\left(k_{1}, k_{2}, k_{3}, k_{4}\right)\right)$, we have

$$
\begin{aligned}
\int_{0}^{t} \lambda_{\min }(K)\|\tilde{e}\|^{2} d t & \leq \int_{0}^{t} \tilde{e}^{T} K \tilde{e} d t \\
& \leq \int_{0}^{t}-\dot{V} d t=V(0)-V(t) \leq V(0),
\end{aligned}
$$

where $\lambda_{\min }(K)$ is the minimum eigenvalue of $K$, so $\widetilde{e}_{1}, \widetilde{e}_{2}, \widetilde{e}_{3}, \widetilde{e}_{4} \in L_{\infty}$; according to Barbalat's Lemma we have

$$
\lim _{t \rightarrow \infty}\|\widetilde{\mathbf{e}}(t)\|=0
$$

The UPS of system (10) and system (11) in the sense of vector function $\boldsymbol{\beta}(t)$ is achieved.

Now we illustrate the effectiveness of the method. Suppose the vector function $\beta(t)=(2 \sin t+\cos 2 t, 32 \sin (3 t)+$ $6,6 \cos t, 2.7 \sin (2 t)+3 \cos t)^{T}$. The "unknown" parameters are $a=36, b=3, c=20, d=1.3, a_{1}=26$, and $b_{1}=14$. Control gain is $\left(k_{1}, k_{2}, k_{3}, k_{4}\right)=(2,3,4,5)$. The scaling matrix is $\mathbf{A}(t)=\operatorname{diag}(\sin 2 t+2,-\cos 3 t-3, \sin t+\cos t+3,2 \sin t-3)$. The initial values of the drive system and response system are $(3,-4,2,2)$ and $(5,7,9,11)$, respectively. The parameter estimate initial values are $\widehat{a}(0)=6, \widehat{b}(0)=-3, \widehat{c}(0)=6$, $\widehat{d}(0)=6, \widehat{a}_{1}(0)=6$, and $\widehat{b}_{1}(0)=3$. Simulation results are as Figures $1,2,3,4,5,6,7$, and 8 .

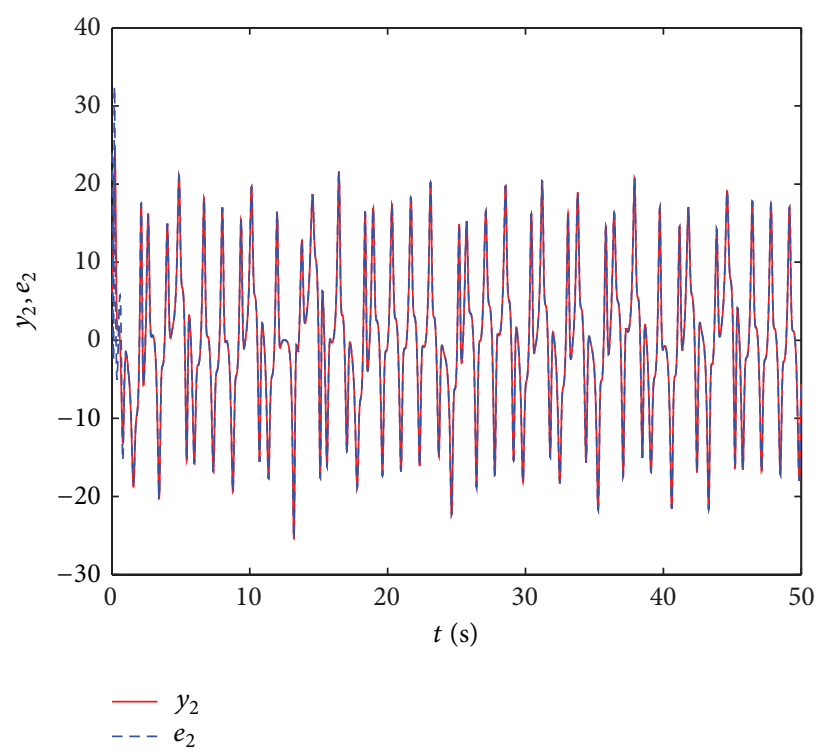

Figure 20: The time evolution of the error $e_{2}$ and the state $y_{2}$.

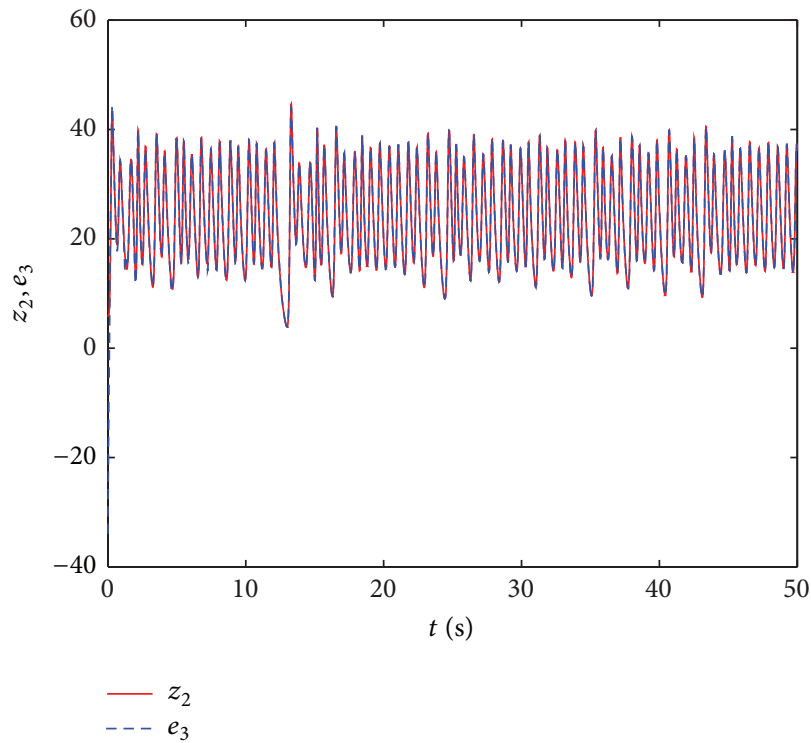

Figure 21: The time evolution of the error $e_{3}$ and the state $z_{2}$.

3.2. UPS in the Sense of a Hyperchaotic System. We take hyperchaotic Wang system [18] as the reference system, which is given by

$$
\begin{gathered}
\dot{x}_{2}=a_{2}\left(-x_{2}+y_{2}\right)+w_{2}, \\
\dot{y}_{2}=c_{2} x_{2}-y_{2}-x_{2} z_{2}, \\
\dot{z}_{2}=x_{2} y_{2}-b_{2} z_{2} \\
\dot{w}_{2}=-y_{2} z_{2}+d_{2} w_{2}
\end{gathered}
$$

where $x_{2}, y_{2}, z_{2}, w_{2}$ are state variables and $a_{2}=10, b_{2}=8 / 3$, $c_{2}=28$, and $d_{2}=-1$ are parameters.

Let $\widetilde{\mathbf{e}}=\left(\widetilde{e}_{1}, \widetilde{e}_{2}, \widetilde{e}_{3}, \widetilde{e}_{4}\right)^{T} \triangleq\left(e_{1}-x_{2}, e_{2}-y_{2}, e_{3}-z_{2}, e_{4}-w_{2}\right)^{T}$. 


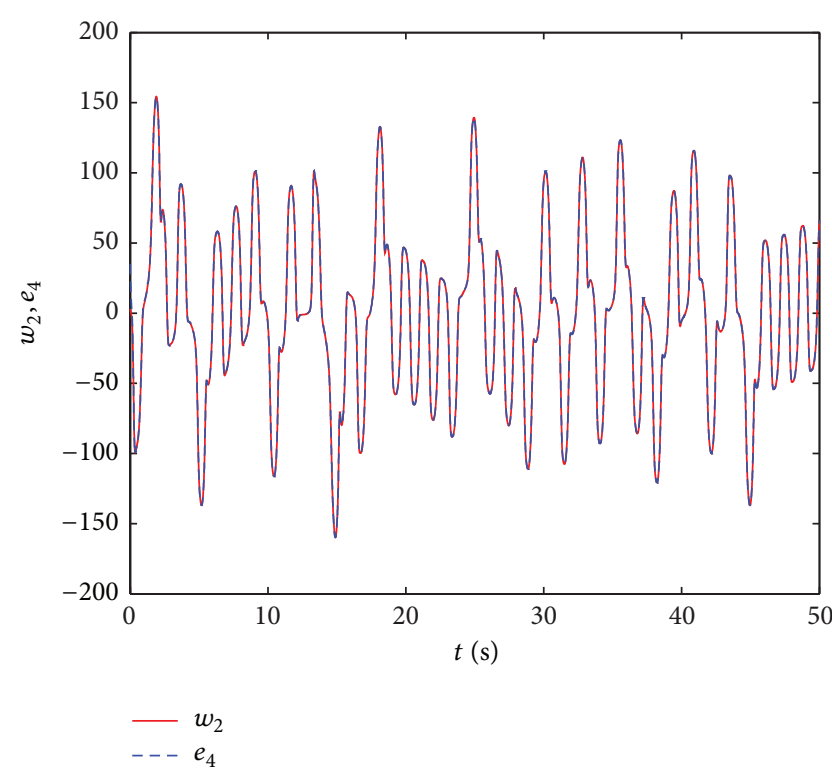

Figure 22: The time evolution of the error $e_{4}$ and the state $w_{2}$.

Theorem 6. For the given scaling matrix $\mathbf{A}(t)$ and the reference system which is hyperchaotic Wang system, the UPS between drive system (10) and response system (11) with unknown parameters will occur by the control $\mathbf{u}=$ $\left(u_{1}, u_{2}, u_{3}, u_{4}\right)$ and parameter update law as follows:

$$
\begin{gathered}
u_{1}=\frac{1}{\alpha_{1}(t)}\left(\hat{a}(y-x)+w-\dot{\alpha}_{1}(t) x_{1}-\alpha_{1}(t) \widehat{a}_{1}\left(y_{1}-x_{1}\right)\right. \\
\left.-\left(a_{2}\left(-x_{2}+y_{2}\right)+w_{2}\right)+k_{1} \widetilde{e}_{1}\right), \\
u_{2}=\frac{1}{\alpha_{2}(t)}\left(-x z+\widehat{c} y-\dot{\alpha}_{2}(t) y_{1}+\alpha_{2}(t) x_{1} z_{1}-\alpha_{2}(t) \widehat{b}_{1} y_{1}\right. \\
\left.-\alpha_{2}(t) w_{1}-\left(c_{2} x_{2}-y_{2}-x_{2} z_{2}\right)+k_{2} \widetilde{e}_{2}\right), \\
u_{3}=\frac{1}{\alpha_{3}(t)}\left(x y-\widehat{b} z-\dot{\alpha}_{3}(t) z_{1}-\alpha_{3}(t) x_{1} y_{1}+3.36 \alpha_{3}(t) z_{1}\right. \\
\left.-\left(x_{2} y_{2}-b_{2} z_{2}\right)+k_{3} \widetilde{e}_{3}\right), \\
u_{4}=\frac{1}{\alpha_{4}(t)}\left(x z+\widehat{d} w-\dot{\alpha}_{4}(t) w_{1}+\alpha_{4}(t) x_{1} z_{1}-\alpha_{4}(t) w_{1}\right. \\
\left.-\left(-y_{2} z_{2}+d_{2} w_{2}\right)+k_{4} \widetilde{e}_{4}\right), \\
\dot{\hat{a}}=(y-x) \widetilde{e}_{1}, \\
\dot{\vec{b}}=-z \widetilde{e}_{3}, \\
\dot{\hat{c}}=y \widetilde{e}_{2}, \\
\dot{\hat{d}}=w \widetilde{e}_{4}, \\
\dot{\hat{a}}=-\alpha_{1}(t)\left(y_{1}-x_{1}\right) \widetilde{e}_{1}, \\
\dot{\hat{b}}=-\alpha_{2}(t) y_{1} \widetilde{e}_{2} .
\end{gathered}
$$

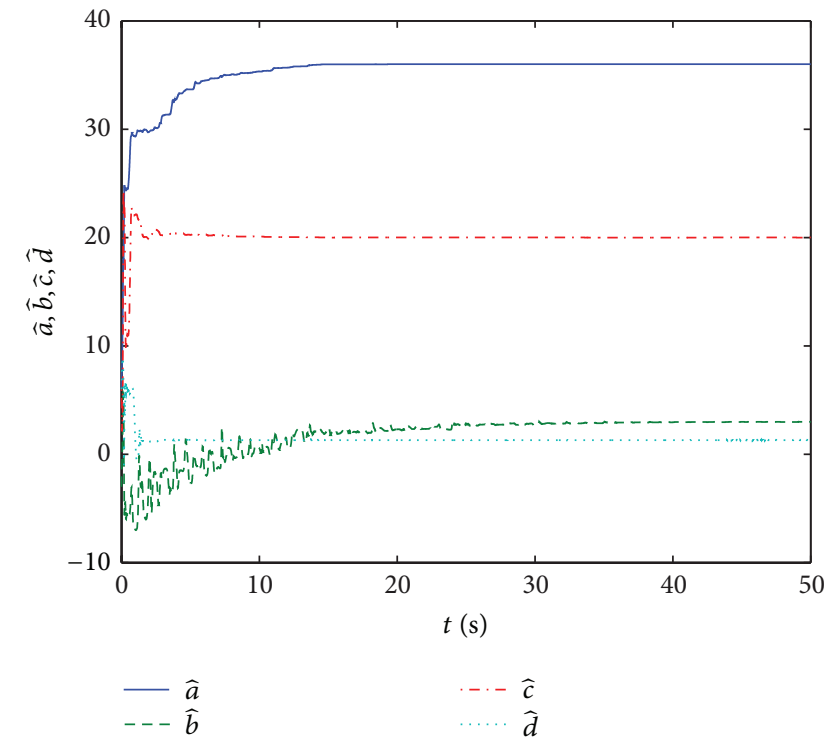

FIgURE 23: The time evolution of the estimated values $\widehat{a}, \widehat{b}, \widehat{c}, \widehat{d}$.

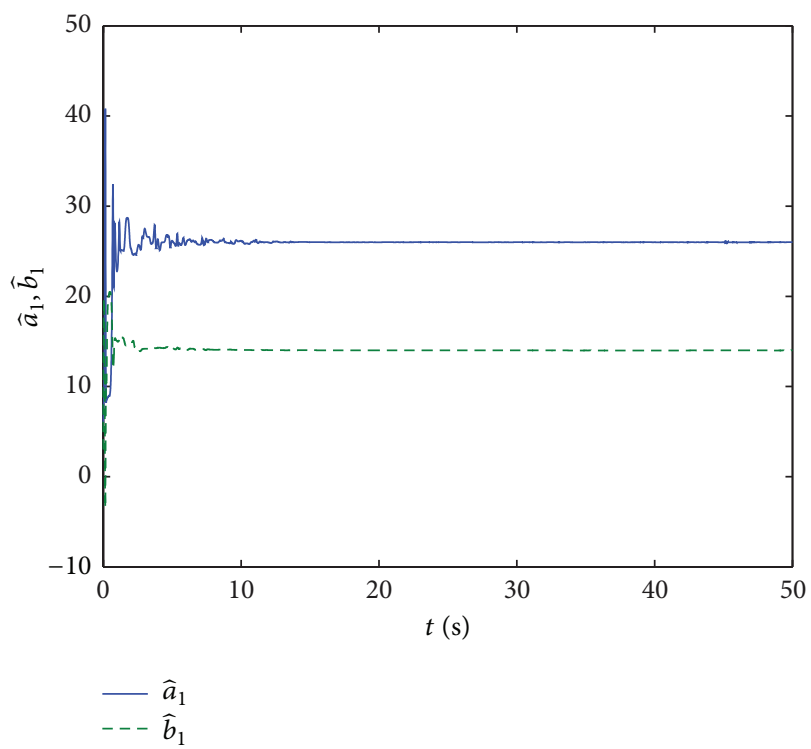

FIGURE 24: The time evolution of the estimated values $\widehat{a}_{1}, \widehat{b}_{1}$.

The proof is similar to Theorem 5 (omitted!).

Now we illustrate the effectiveness of this method. Suppose the initial value of hyperchaotic Wang is $(1,6,6,3)$. The "unknown" parameters are $a=36, b=3, c=20, d=1.3$, $a_{1}=26$, and $b_{1}=14$. Control gain is $\left(k_{1}, k_{2}, k_{3}, k_{4}\right)=$ $(12,13,14,15)$. The scaling matrix is $\mathbf{A}(t)=\operatorname{diag}(\sin 2 t+$ $2,-\cos 3 t-3, \sin t+\cos t+3,2 \sin t-3)$. The initial values of the drive system and response system are $(3,-4,2,2)$ and $(5,7,9,11)$, respectively. The parameter estimate initial values are $\widehat{a}(0)=6, \widehat{b}(0)=-3, \widehat{c}(0)=6, \widehat{d}(0)=6, \widehat{a}_{1}(0)=6$, and $\widehat{b}_{1}(0)=3$. Simulation results are as Figures $9,10,11,12,13,14$, 15 , and 16 . 


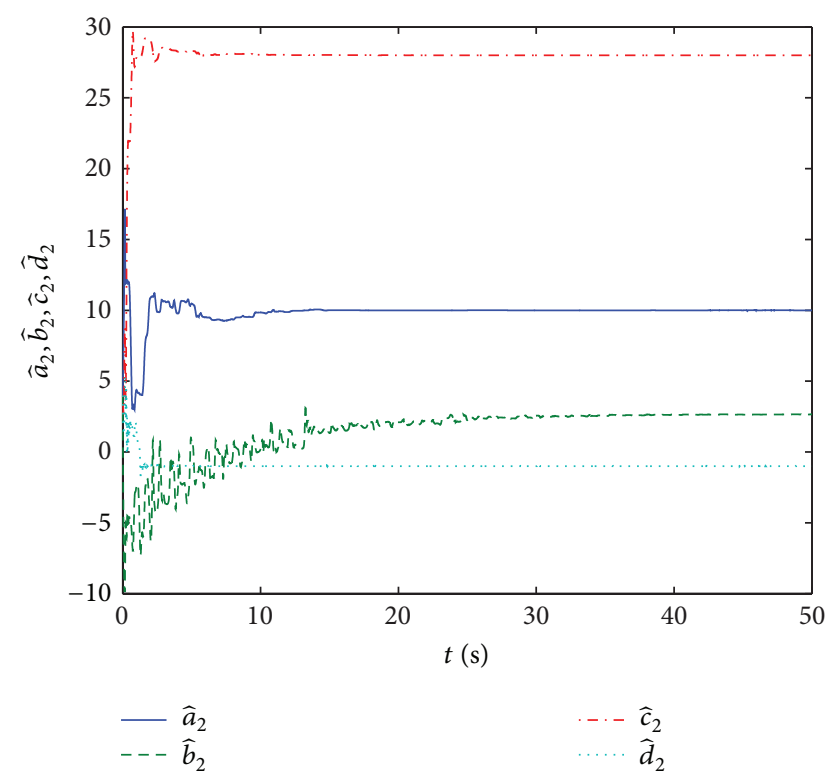

FIGURE 25: The time evolution of the estimated values $\widehat{a}_{2}, \widehat{b}_{2}, \widehat{c}_{2}, \widehat{d}_{2}$.

3.3. UPS in the Sense of a Hyperchaotic Wang System with Unknown Parameters. We still use hyperchaotic Wang system as the reference system, but the parameters $a_{2}, b_{2}, c_{2}, d_{3}$ are uncertain. Suppose the estimated values of $a_{2}, b_{2}, c_{2}, d_{3}$ are $\widehat{a}_{2}, \widehat{b}_{2}, \widehat{c}_{2}, \widehat{d}_{2}$, respectively; the parameter errors are $\left(\widetilde{a}_{2}, \widetilde{b}_{2}, \widetilde{c}_{2}, \widetilde{d}_{2}\right)=\left(\widehat{a}_{2}-a_{2}, \widehat{b}_{2}-b_{2}, \widehat{c}_{2}-c_{2}, \widehat{d}_{2}-d_{2}\right)$. Let $\widetilde{\mathbf{e}}=$ $\left(\widetilde{e}_{1}, \widetilde{e}_{2}, \widetilde{e}_{3}, \widetilde{e}_{4}\right)^{T} \triangleq\left(e_{1}-x_{2}, e_{2}-y_{2}, e_{3}-z_{2}, e_{4}-w_{2}\right)^{T}$.

Theorem 7. For the given scaling matrix $\mathbf{A}(t)$ and the reference system which is hyperchaotic Wang system with unknown parameters, the UPS between drive system (10) and response system (11) with unknown parameters will occur by the control $\mathbf{u}=\left(u_{1}, u_{2}, u_{3}, u_{4}\right)$ and parameter update law as follows:

$$
\begin{gathered}
u_{1}=\frac{1}{\alpha_{1}(t)}\left(\widehat{a}(y-x)+w-\dot{\alpha}_{1}(t) x_{1}-\alpha_{1}(t) \widehat{a}_{1}\left(y_{1}-x_{1}\right)\right. \\
\left.\quad-\widehat{a}_{2}\left(-x_{2}+y_{2}\right)-w_{2}+k_{1} \widetilde{e}_{1}\right), \\
\begin{aligned}
u_{2}=\frac{1}{\alpha_{2}(t)}( & -x z+\widehat{c} y-\dot{\alpha}_{2}(t) y_{1}+\alpha_{2}(t) x_{1} z_{1}-\alpha_{2}(t) \widehat{b}_{1} y_{1} \\
& \left.-\alpha_{2}(t) w_{1}-\widehat{c}_{2} x_{2}+y_{2}+x_{2} z_{2}+k_{2} \widetilde{e}_{2}\right), \\
u_{3}=\frac{1}{\alpha_{3}(t)}( & x y-\widehat{b} z-\dot{\alpha}_{3}(t) z_{1}-\alpha_{3}(t) x_{1} y_{1}+3.36 \alpha_{3}(t) z_{1} \\
& \left.-x_{2} y_{2}+\widehat{b}_{2} z_{2}+k_{3} \widetilde{e}_{3}\right), \\
u_{4}=\frac{1}{\alpha_{4}(t)}( & x z+\widehat{d} w-\dot{\alpha}_{4}(t) w_{1}+\alpha_{4}(t) x_{1} z_{1}-\alpha_{4}(t) w_{1} \\
& \left.+y_{2} z_{2}-\widehat{d}_{2} w_{2}+k_{4} \widetilde{e}_{4}\right),
\end{aligned}
\end{gathered}
$$

$$
\begin{gathered}
\dot{\hat{a}}=(y-x) \widetilde{e}_{1}, \\
\dot{\hat{b}}=-z \widetilde{e}_{3}, \\
\dot{\hat{c}}=y \widetilde{e}_{2}, \\
\dot{\hat{d}}=w \widetilde{e}_{4}, \\
\dot{\hat{a}}_{1}=-\alpha_{1}(t)\left(y_{1}-x_{1}\right) \widetilde{e}_{1}, \\
\dot{\hat{b}}_{1}=-\alpha_{2}(t) y_{1} \widetilde{e}_{2}, \\
\dot{\hat{a}}_{2}=-\left(-x_{2}+y_{2}\right) \widetilde{e}_{1}, \\
\dot{\hat{b}}_{2}=z_{2} \widetilde{e}_{3}, \\
\dot{\hat{c}}_{2}=-x_{2} \widetilde{e}_{2}, \\
\dot{\hat{d}}_{2}=-w_{2} \widetilde{e}_{4} .
\end{gathered}
$$

Proof. Choose the following Lyapunov function:

$$
\begin{gathered}
V=\frac{1}{2}\left(\widetilde{\mathbf{e}}^{T} \widetilde{\mathbf{e}}+\widetilde{a}^{2}+\widetilde{b}^{2}+\widetilde{c}^{2}+\widetilde{d}^{2}+\widetilde{a}_{1}^{2}\right. \\
\left.+\widetilde{b}_{1}^{2}+\widetilde{a}_{2}^{2}+\widetilde{b}_{2}^{2}+\widetilde{c}_{2}^{2}+\widetilde{d}_{2}^{2}\right) .
\end{gathered}
$$

Then the derivative of $V$ is given by

$$
\begin{aligned}
& \dot{V}=\frac{1}{2}\left(2 \widetilde{\mathbf{e}}^{T} \dot{\overrightarrow{\mathbf{e}}}+2 \widetilde{a} \dot{\vec{a}}+2 \widetilde{b} \dot{\widetilde{b}}+2 \dot{\widetilde{c}} \dot{\vec{c}}+2 \widetilde{\overrightarrow{d d}}+2 \widetilde{a}_{1} \dot{\tilde{a}}_{1}+2 \widetilde{b}_{1} \dot{\widetilde{b}}_{1}\right. \\
& \left.+2 \widetilde{a}_{2} \dot{\vec{a}}_{2}+2 \widetilde{b}_{2} \dot{\widetilde{b}}_{2}+2 \widetilde{c}_{2} \dot{\vec{c}}_{2}+2 \widetilde{d}_{2} \dot{\tilde{d}}_{2}\right) \\
& =\widetilde{\mathbf{e}}^{T} \dot{\widetilde{\mathbf{e}}}+\tilde{a} \dot{\vec{a}}+\tilde{b} \dot{\vec{b}}+\dot{\vec{c}} \dot{\vec{c}}+\dot{\widetilde{d}}+\widetilde{a}_{1} \dot{\tilde{a}}_{1}+\widetilde{b}_{1} \dot{\vec{b}}_{1} \\
& +\tilde{a}_{2} \dot{\tilde{a}}_{2}+\widetilde{b}_{2} \dot{\vec{b}}_{2}+\widetilde{c}_{2} \dot{\vec{c}}_{2}+\widetilde{d}_{2} \dot{\vec{d}}_{2} \\
& =\widetilde{e}_{1}\left(a(y-x)+w-\dot{\alpha}_{1} x_{1}-\alpha_{1} a_{1}\left(y_{1}-x_{1}\right)\right. \\
& \left.-\alpha_{1} u_{1}-a_{2}\left(-x_{2}+y_{2}\right)-w_{2}\right) \\
& +\tilde{e}_{2}\left(-x z+c y-\dot{\alpha}_{2} y_{1}-\alpha_{2}\left(b_{1} y_{1}-x_{1} z_{1}+w_{1}\right)\right. \\
& \left.-\alpha_{2} u_{2}-c_{2} x_{2}+y_{2}+x_{2} z_{2}\right) \\
& +\widetilde{e}_{3}\left(x y-b z-\dot{\alpha}_{3} z_{1}-\alpha_{3}\left(x_{1} y_{1}-c_{1} z_{1}\right)\right. \\
& \left.-\alpha_{3} u_{3}-x_{2} y_{2}+b_{2} z_{2}\right) \\
& +\widetilde{e}_{4}\left(x z+d w-\dot{\alpha}_{4} w_{1}-\alpha_{4}\left(-x_{1} z_{1}+w_{1}\right)\right. \\
& \left.-\alpha_{4} u_{4}+y_{2} z_{2}-d_{2} w_{2}\right) \\
& +\tilde{a} \dot{\tilde{a}}+\tilde{b} \dot{\vec{b}}+\dot{\vec{c}} \dot{\vec{c}}+\tilde{\overrightarrow{d d}}+\widetilde{a}_{1} \dot{\tilde{a}}_{1}+\widetilde{b}_{1} \dot{\vec{b}}_{1} \\
& +\widetilde{a}_{2} \dot{\tilde{a}}_{2}+\widetilde{b}_{2} \dot{\vec{b}}_{2}+\widetilde{c}_{2} \dot{\tilde{c}}_{2}+\widetilde{d}_{2} \dot{\vec{d}}_{2}
\end{aligned}
$$

With the controls (22) and the parameter update laws (23) and (24), we have

$$
\dot{V}=-k_{1} \widetilde{e}_{1}^{2}-k_{2} \widetilde{e}_{2}^{2}-k_{3} \widetilde{e}_{3}^{2}-k_{4} \widetilde{e}_{4}^{2} .
$$


Because $\dot{V}$ is negative simidefinite, $\widetilde{e}_{1}, \widetilde{e}_{2}, \widetilde{e}_{3}, \widetilde{e}_{4} \in L_{\infty}$, $\tilde{a}, \widetilde{b}, \widetilde{c}, \widetilde{d}, \widetilde{a}_{1}, \widetilde{b}_{1}, \widetilde{a}_{2}, \widetilde{b}_{2}, \widetilde{c}_{2}, \widetilde{d}_{2} \in L_{\infty}$. From the definition of $\widetilde{\mathbf{e}}$, we know $\dot{\tilde{e}}_{1}, \dot{\tilde{e}}_{2}, \dot{\tilde{e}}_{3}, \dot{\tilde{e}}_{4} \in L_{\infty}$. From $\dot{V}=-k_{1} \widetilde{e}_{1}^{2}-k_{2} \widetilde{e}_{2}^{2}-k_{3} \widetilde{e}_{3}^{2}-k_{4} \widetilde{e}_{4}^{2}=$ $-\widetilde{e}^{T} K \widetilde{e}\left(K=\operatorname{diag}\left(k_{1}, k_{2}, k_{3}, k_{4}\right)\right)$, we have

$$
\begin{aligned}
\int_{0}^{t} \lambda_{\min }(K)\|\tilde{e}\|^{2} d t & \leq \int_{0}^{t} \tilde{e}^{T} K \widetilde{e} d t \\
& \leq \int_{0}^{t}-\dot{V} d t=V(0)-V(t) \leq V(0),
\end{aligned}
$$

where $\lambda_{\min }(K)$ is the minimum eigenvalue of $K$, so $\widetilde{e}_{1}, \widetilde{e}_{2}, \widetilde{e}_{3}, \widetilde{e}_{4} \in L_{\infty}$; according to Barbalat's Lemma we have

$$
\lim _{t \rightarrow \infty}\|\widetilde{\mathbf{e}}(t)\|=0 \text {. }
$$

The UPS of system (10) and system (11) in the sense of hyperchaotic Wang system (19) with uncertain parameters is achieved.

Now we illustrate the effectiveness of this method. Suppose the initial value of hyperchaotic Wang system is $(1,6,6,3)$. The "unknown" parameters are $a=36, b=3$, $c=20, d=1.3, a_{1}=26, b_{1}=14, a_{2}=10, b_{2}=8 / 3$, $c_{2}=28$, and $d_{2}=-1$. Control gain is $\left(k_{1}, k_{2}, k_{3}, k_{4}\right)=$ $(12,13,14,15)$. The scaling matrix is $\mathbf{A}(t)=\operatorname{diag}(\sin 2 t+$ $2,-\cos 3 t-3, \sin t+\cos t+3,2 \sin t-3)$. The initial values of the drive system and the response system are $(3,-4,2,2)$ and $(5,7,9,11)$, respectively. The parameter estimate initial values are $\widehat{a}(0)=6, \widehat{b}(0)=-3, \widehat{c}(0)=6, \widehat{d}(0)=6, \widehat{a}_{1}(0)=6$, $\widehat{b}_{1}(0)=3, \widehat{a}_{2}(0)=4, \widehat{b}_{2}(0)=4, \widehat{c}_{2}(0)=4$, and $\widehat{d}_{2}(0)=4$. Simulation results are as Figures 17, 18, 19, 20, 21, 22, 23, 24, and 25.

From the above analysis, we find that, in the form, the adaptive laws equations (13), (21), and (23) corresponding three kinds of reference systems are the same.

\section{Conclusion}

In this paper, we have defined universal projective synchronization. For different hyperchaotic systems with uncertain parameters, we have given adaptive control method such that UPS has occured and the parameters have been determined. Three kinds of reference systems have been concerned and we find that, in the form, the adaptive laws are the same. Numerical simulations have verified the effectiveness of the scheme.

\section{Conflict of Interests}

The authors declare that there is no conflict of interests regarding the publication of this paper.

\section{Acknowledgments}

This work is supported partly by the National Natural Science Foundation of China (Grant no. 61374118), the program of Yunnan Provincial Science and Technology Department (Grant no. 2013FD046), and Youth Foundation of Qujing Normal University (Grant no. 2008QN034).

\section{References}

[1] L. M. Pecora and T. L. Carroll, "Synchronization in chaotic systems," Physical Review Letters, vol. 64, no. 8, pp. 821-824, 1990.

[2] N. F. Rulkov, M. M. Sushchik, L. S. Tsimring, and H. D. I. Abarbanel, "Generalized synchronization of chaos in directionally coupled chaotic systems," Physical Review E, vol. 51, no. 2, pp. 980-994, 1995.

[3] Q. Jia, "Projective synchronization of a new hyperchaotic Lorenz system," Physics Letters A, vol. 370, no. 1, pp. 40-45, 2007.

[4] L. Y. Cao and Y. C. Lai, "Antiphase synchronism in chaotic systems," Physical Review E, vol. 58, no. 1, pp. 382-386, 1998.

[5] R. Mainieri and J. Rehacek, "Projective synchronization in three-dimensional chaotic systems," Physical Review Letters, vol. 82, no. 15, pp. 3042-3045, 1999.

[6] J. Yan and C. Li, "Generalized projective synchronization of a unified chaotic system," Chaos, Solitons \& Fractals, vol. 26, no. 4, pp. 1119-1124, 2005.

[7] G. H. Li, "Modified projective synchronization of chaotic system," Chaos, Solitons and Fractals, vol. 32, no. 5, pp. 17861790, 2007.

[8] Y. Chen and X. Li, "Function projective synchronization between two identical chaotic systems," International Journal of Modern Physics C, vol. 18, no. 5, pp. 883-888, 2007.

[9] X. Tang, J. Lu, and W. Zhang, "The funciton projective synchronization of chaotic system using backstepping design," Journal of Dynimics and Control, vol. 5, pp. 216-219, 2007.

[10] H. Du, Q. Zeng, and C. Wang, "Function projective synchronization of different chaotic systems with uncertain parameters," Physics Letters A, vol. 372, no. 33, pp. 5402-5410, 2008.

[11] H. Du, Q. Zeng, and C. Wang, "Modified function projective synchronization of chaotic system," Chaos, Solitons and Fractals, vol. 42, no. 4, pp. 2399-2404, 2009.

[12] J. F. Li and N. Li, "Modified function projective synchronization of a class of chaotic systems," Acta Physica Sinica, vol. 60, no. 8, Article ID 080507, 2011.

[13] G. Fu, "Robust adaptive modified function projective synchronization of different hyperchaotic systems subject to external disturbance," Communications in Nonlinear Science and Numerical Simulation, vol. 17, no. 6, pp. 2602-2608, 2012.

[14] J. A. Wang and H. P. Liu, "Adaptive modified function projective synchronization of different hyperchaotic systems," Acta Physica Sinica, vol. 59, no. 4, pp. 2265-2271, 2010.

[15] T. L. Liao and S. H. Lin, "Adaptive control and synchronization of Lorenz systems," Journal of the Franklin InstituteEngineering and Applied Mathematics, vol. 336, no. 6, pp. 925937, 1999.

[16] B. Zhang, H. Li, and H. Guo, "A new hyperchaotic system and the synchronization using active variable universe adaptive fuzzy controller," Mathematical Problems in Engineering, vol. 2013, Article ID 763219, 10 pages, 2013.

[17] A. Chen, J. Lu, J. Lü, and S. Yu, "Generating hyperchaotic Lü attractor via state feedback control," Physica A, vol.364, pp. 103110, 2006.

[18] X. Wang and M. Wang, "A hyperchaos generated from Lorenz system," Physica A, vol. 387, no. 14, pp. 3751-3758, 2008. 


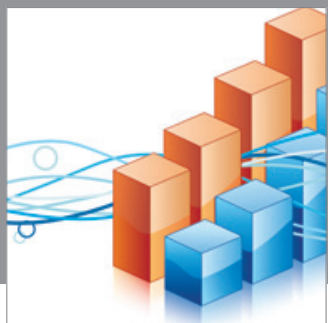

Advances in

Operations Research

mansans

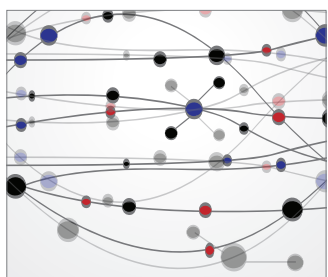

The Scientific World Journal
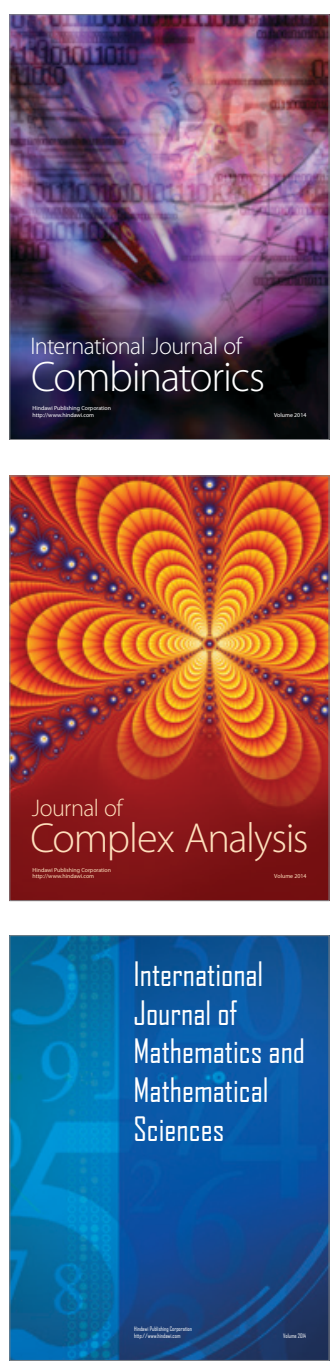
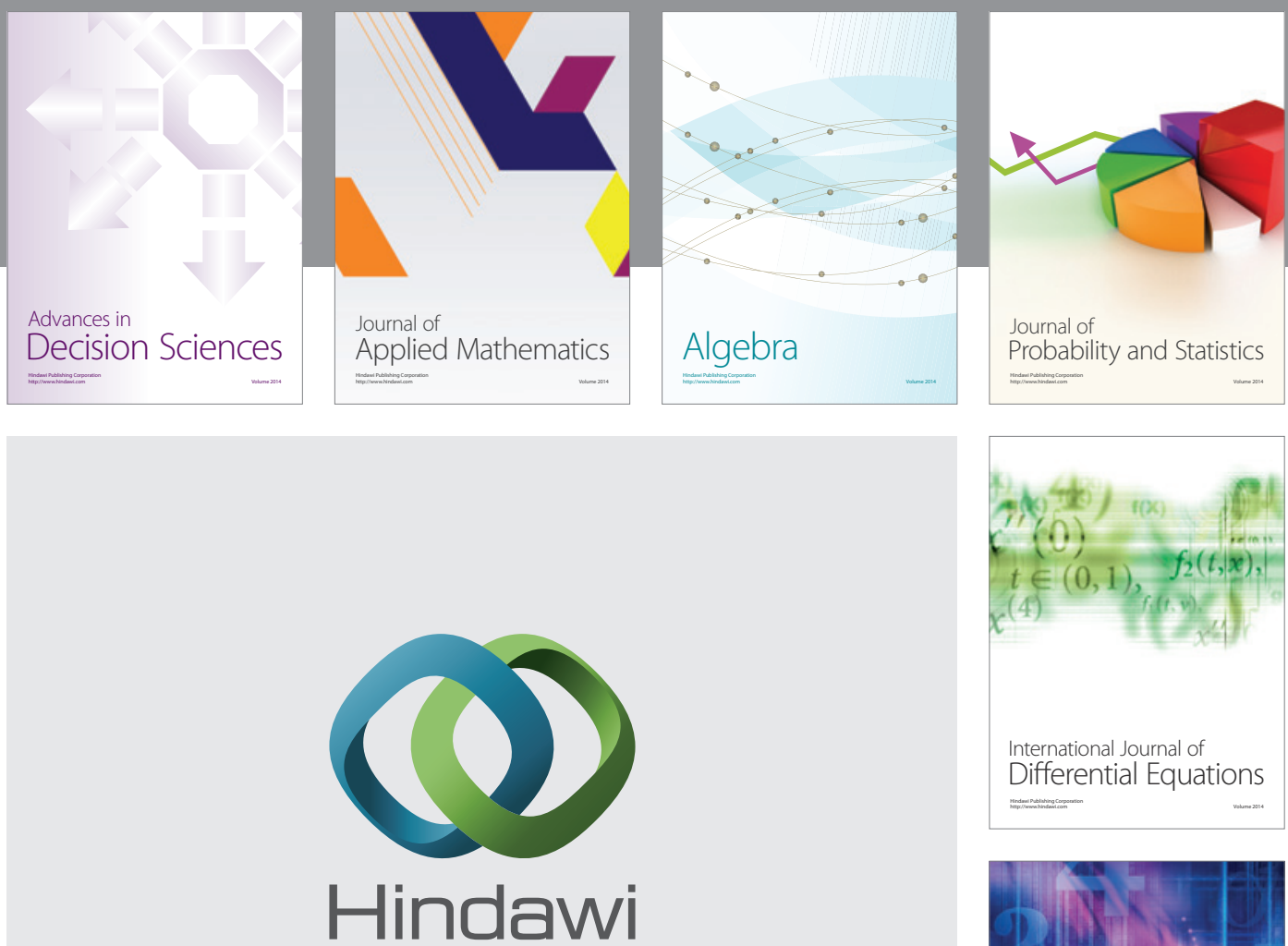

Submit your manuscripts at http://www.hindawi.com
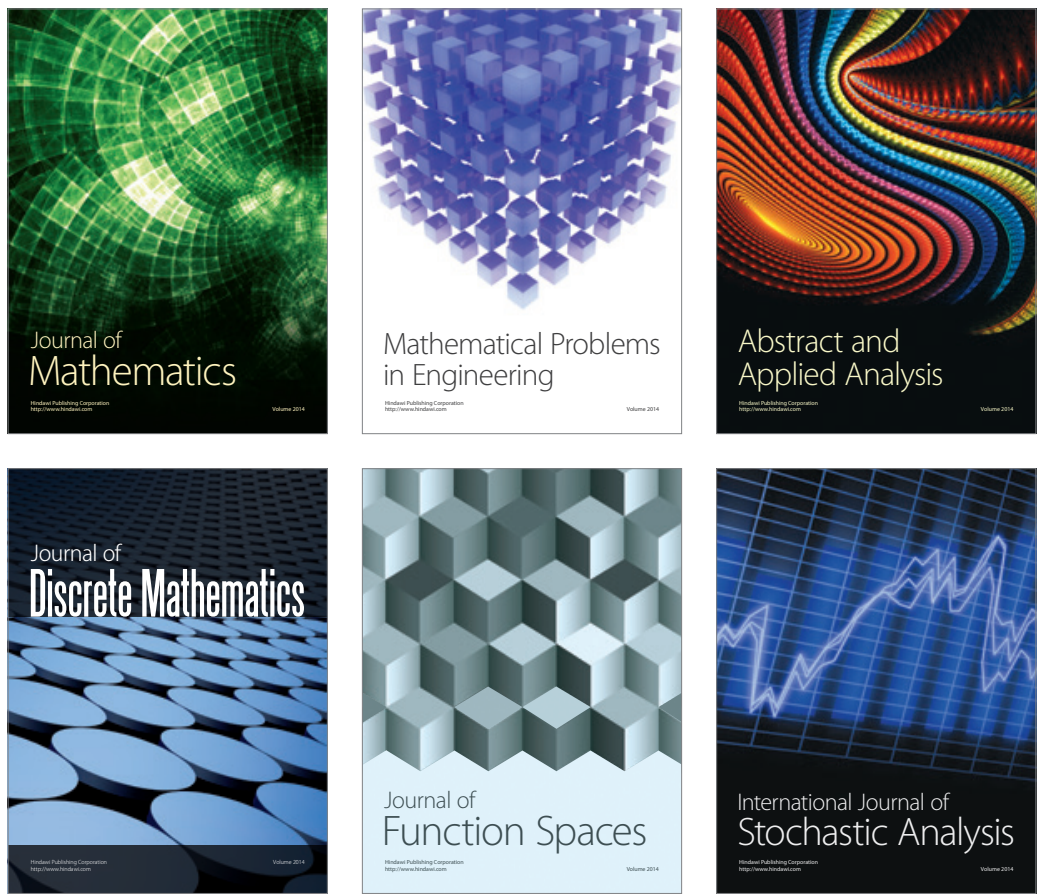

Journal of

Function Spaces

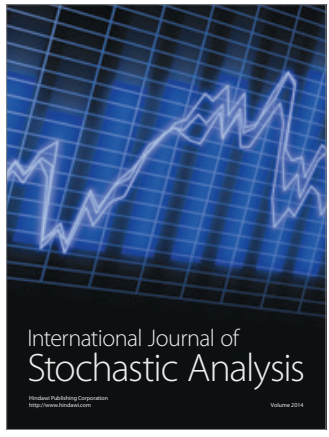

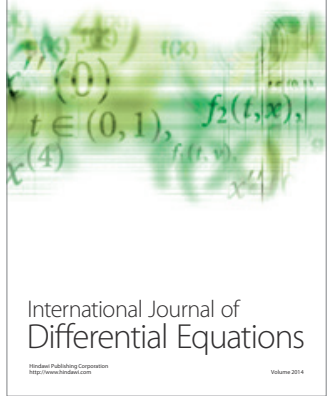
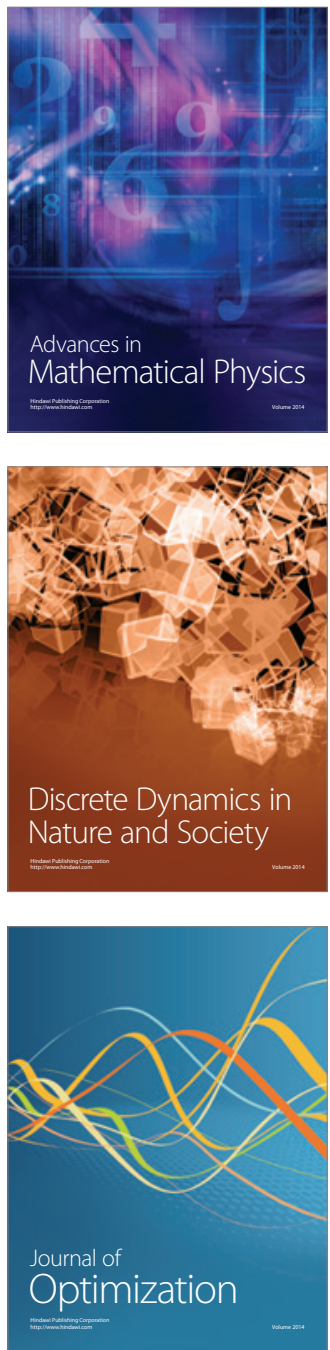